\title{
Rapid Assembly Processes of Ordered Inorganic/organic Nanocomposites
}

\author{
Chang-An Wang ${ }^{1}$, Huirong Le ${ }^{2}$ and Yong Huang ${ }^{1}$ \\ ${ }^{1}$ Tsinghua University, Beijing 100084, P.R. China \\ 2 University of Dundee, Dundee DD1 4HN, United Kingdom
}

\section{Introduction}

The material of seashell nacre is of great interest to material scientists due to its superior mechanical properties and unique bio-compatibility. Natural seashell nacre consists of about $95 \%$ of aragonite (a mineral form of $\mathrm{CaCO}_{3}$ ) and a few percent of biological macromolecules; yet its work of fracture is about three orders of magnitude higher than its mineral constituency. The superior strength and toughness of seashell nacre are attributed to the robust nanostructure in which the protein collagen layers (10-50 nm thick) and aragonite tablets (200-900 nm thick) form an ordered brick-and-mortar structure. The macromolecules act as strong adhesives while the aragonite tablets as the rigid building blocks (Smith et al., 1999). The structure of seashell nacre has inspired material scientists to design and develop various advanced biomimetic materials in the last two decades.

A macroscopic analogue of nacre structure led to the development of laminated silicon nitride ceramic composites which exhibit superior toughness to monolithic ceramics (Clegg et al., 1990; Wang et al., 2002), however, the laminated structure in micrometer scale may not have the same performance as the one with nano-scale laminated structure. In recent years, there is an increasing interest in the development of biomimetic organic/inorganic nanocomposites. These new materials hold promise for high performance orthopaedic materials, chemical sensors and photonic functional materials.

The formation of seashell nacre is a slow process in nature. Seashell absorbs mineral elements and organic matters in seawater to form inorganic nano-platelets and macromolecules, which deposit alternately to form compact brick-and-mortar structure of nacre. There are several approaches to mimic this process. One approach is called layer-bylayer assembly, by which ultrathin films of multilayered structure were prepared by sequential adsorption of a cationic polyelectrolyte and individual platelets of the negatively charged silicate mineral (Kleinfeld \& Ferguson, 1994). Kleinfeld and Ferguson reported the incorporation of these clay sheets into thin films by the electrostatic layer-by-layer assembly (LBL). The structural investigation of film thickness and interlayer spacing of clay particles was accomplished by Kotov et al (Kotov et al., 1997) through a series of surface-sensitive techniques. The determination of the mechanism of the layer-by-layer alternating selfassembly of the clay multilayers was reported (Kotov et al., 1997; Kleinfeld \& Ferguson, 
1996). Fan et al. (Fan et al., 2002) fabricated a sexithiophene/montmorillonite multilayered film by the electrostatic layer-by-layer deposition technique, which was considered to be the first system for the preparation of sexithiophene ultrathin films. Tang et al. (Tang et al., 2003) reported a method to reproduce an analogue of nacre by sequential deposition of polyelectrolytes and clays, of which mechanical properties approached that of nacre or lamella bones. However, since LBL assembly process requires many repeated steps to build up a practical film thickness, for example, a thin film of a thickness of $5 \mu \mathrm{m}$ took more than 2 days to form, it is a very slow process and can only produce ultrathin films. Lu et al. (Lu et al., 1997) reported a rapid way, the evaporation-induced self-assembly, to prepare orderly laminated organic-inorganic composites. Huang et al. (Huang et al., 1998)] also employed this evaporation-induced self-assembly process to prepare continuous highly ordered lamellar-phase mesostructured thin films. By this method, however, high uniformity and stability could be obtained only for thin films (Sellinger et al., 1998; Deville et al., 2006). Deville et al. (Deville et al., 2006) developed a freeze-casting process to fabricate porous, layered materials. This process offers a large number of material combinations and a wide range of dimension control. However, this method could not be extended into nanoscale. The organic content in the composites obtained by the existing methods is much higher than that contained in natural nacres. Moreover, the product is intrinsically porous and needs to be filled with a second phase to make a composite material.

As mentioned above, the deposition speed of current technologies is not efficient to fabricate orderly bulk materials. This chapter will introduce a number of efficient processes to generate ordered inorganic/organic nanocomposites currently being developed. Section 1 will describe an efficient centrifugal deposition and in-situ polymerization process to fabricate polyimide/montmorillonite (PI/MMT) nanocomposites (Chen et al., 2008). This process results in an ordered nanocomposite with alternating organic and inorganic layers and mechanical properties comparable to that of lamella bones. Section 2 will introduce an electrophoretic deposition process and in-situ polymerization to fabircate polyacrylamidemontmorillonite (PAM/MMT) composite (Long et al., 2007). The process involves preparation of water based suspension, electrophoretic deposition and ultraviolet initiated polymerization. This process can produce nano-laminated composite films, similar to nacre both in structure and organic content. The composite film displays significant enhancement in Young's modulus and hardness, compared to that of pure montmorillonite films. Section 3 will put forward a special assembly method - hydrothermal-electrophoretic assembly to prepare acrylic anodic electrophoretic resin (AAER)/montmorillonite nanocomposite that mimics nacre, both in structure and composition. The two-step assembly process includes intercalation of polymer into interlayer space of montmorillonite by hydrothermal process and the subsequent electrophoretic deposition. By constructing brick-and-mortar nanostructure, Young's modulus of the composite film is effectively enhanced even when organic content is low (Lin et al., 2008). Section 4 will present an electrophoretic deposition of aqueous suspension containing montmorillonite nano-plates and polyelectrolyte such as acrylic anodic electrophoretic resin (AAER) or cathodic electrophoretic acrylic resin (CEAR) (Lin et al., 2008; Chen et al., in press). The composite obtained has good uniformity and significant improvement in Young's modulus and strength over the polymer substrate. 


\section{Efficient centrifugal deposition and in-situ polymerization process to fabricate polyimide/montmorillonite (PI/MMT) Nanocomposites}

In this section, an efficient centrifugal deposition and in-situ polymerization process is proposed to fabricate bulk-form polyimide/montmorillonite (PI/MMT) nanocomposites. Samples with thickness of $10-200 \mu \mathrm{m}$ were produced in a very short time. This process resulted in an ordered nanostructure with alternating organic and inorganic layers and mechanical properties comparable to that of lamella bones.

\subsection{Experimental procedure}

\subsubsection{Composite preparation}

The raw montmorillonite, a $\mathrm{Ca}^{2+}$-type montmorillonite, was provided by Deshun Montmorillonite Plant, Shunping County, Hebei Province, China. The MMT powder was first treated with sodium salt solution in hot water at $75{ }^{\circ} \mathrm{C}$ and washed to remove $\mathrm{Ca}^{2+}$, to improve the organic cation exchange capacity (CEC). The MMT powder was then treated with cetyltrimethyl ammonium chloride (CTAC) under the same conditions, to convert the normally hydrophilic silicate to hydrophobic one, which is called organic-MMT. The organic-MMT platelets were thoroughly dispersed using strong ultrasonic agitator in $\mathrm{N}, \mathrm{N}$ dimethylacetamide solution, then added with 4,4'-diaminodiphenyl ether and pyromellitic dianhydride and finally stirred strongly at $30^{\circ} \mathrm{C}$ for 4 hours. MMT platelets covered by imide monomers described above were subsequently deposited onto glass substrate by centrifugation at a speed of $3000 \mathrm{rpm}$ for about $15 \mathrm{~min}$. Samples were also prepared by deposition under normal gravity for comparison. All the samples were heat-treated at $300{ }^{\circ} \mathrm{C}$ for 1 hour to facilitate polymerization of imide monomers. The glass slide carrying PI/MMT was immersed into $0.5 \mathrm{wt} \% \mathrm{HF}$ solution so that the composite slices could be easily peeled off from the glass substrate.

\subsubsection{Characterization}

The montmorillonite platelets and microstructure of PI/MMT composites were examined using a field emission scanning electron microscopy (JEOL, JSM-6400, Japan) with an estimated resolution of about $5 \mathrm{~nm}$. X-ray diffraction was taken on D8-Discover machine (Bruker Co., Germany) to examine the ordered laminated structure. FTIR absorbency (SYSTEM 2000, USA) and TGA (SETARAM TGA92, France) were used to determine the formation and content of organic compounds in the organic-inorganic composites. The tensile properties of the specimens were carried under custom-made testing device at a constant tensile speed of $0.001 \mathrm{~mm} / \mathrm{min}$, and the whole process was recorded automatically. Nanoindentations (Berkovich tip, radius of curvature $50 \mathrm{~nm}$ ) were also taken to determine the hardness and Young's modulus. Surface topography of PI/MMT slice was measured by AFM (MI, Picoscan, USA).

\subsection{Results and discussions}

Montmorillonite is a naturally occurring phyllosilicate, which has a similar layered and crystalline structure as talc and mica but with a different layer charge. The MMT crystal lattice consists of a central octahedral sheet of $[\mathrm{Al}, \mathrm{O}, \mathrm{OH}]$ inserted between two $\left[\mathrm{SiO}_{4}\right]$ 
tetrahedral sheets (in such a way that the oxygen ions in the octahedral sheet also belong to the silica tetrahedra). The MMT's layered structure can be easily delaminated into platelets with a thickness about one to several nanometers and a length from $100 \mathrm{~nm}$ to several micrometers by some specific methods. Fig. 1 shows the well-dispersed and separated MMT platelets used hereby.

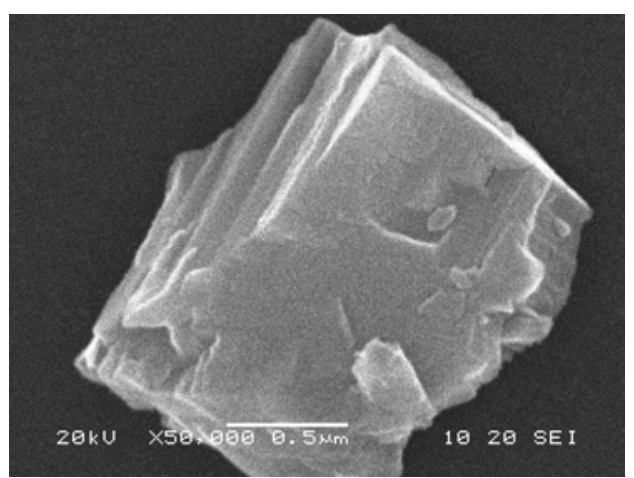

(a)

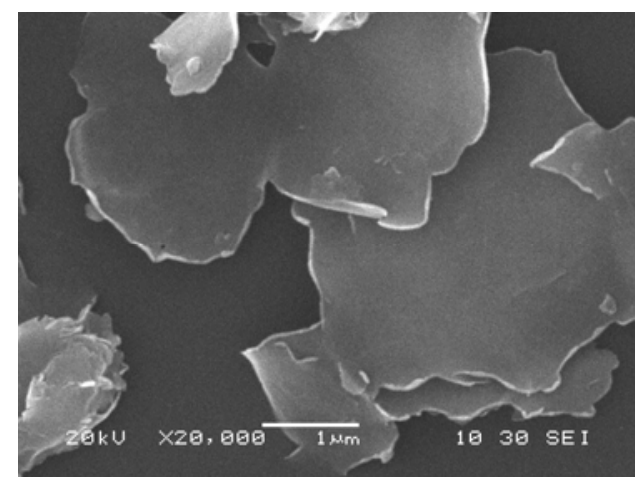

(b)

Fig. 1. (a) Stacks of platelets in original montmorillonite (b) exfoliation of the clay after organic conversion and strong sonication.

It is well known that organic ammonium salts can exchange ion with MMT to form organically modified MMT, which converts MMT from hydrophilic to hydrophobic. The organically modified MMT can be easily dispersed in water solution and absorb organic molecules in the solution. This provides a way to combine inorganic and organic components at molecular level. In the present work, the commercial MMT was treated in an organic ammonium chloride solution and dispersed in water solutions of imide monomers. The imide monomers grafted on the MMT platelet surfaces were polymerized in-situ to form polyimide (PI) macromolecules. X-ray diffraction (XRD) and infrared spectroscopy (IR) analysis (Fig. 2) showed that PI macromolecules were successfully inserted into the sodium interlayer of MMT nano-platelets to form intercalated composite. Organic ammonium chloride plays an important role in this process because it enlarges the d-spacing between adjacent montmorillonite platelets (from $1.244 \mathrm{~nm}$ to $1.842 \mathrm{~nm}$ ). The adsorption of imide monomers and formation of PI macromolecules further expand the distance between MMT layers (from $1.842 \mathrm{~nm}$ to $2.044 \mathrm{~nm}$ ). The results of infrared absorption spectra also indicate that the inorganic "bricks" (MMT platelets) and organic "mortar" (PI) are tightly combined together after the polymerization reaction. The nanostructure of PI/MMT composite system is similar to that of seashell nacre due to the characteristics of the inorganic and organic components.

Thermal gravimetric analysis (Fig. 3) showed the loss of organic compound when artificial nacre was heated to high temperature. There is a large weight loss around $300{ }^{\circ} \mathrm{C}$ due to the removal of free PI macromolecules surrounding the intercalated particle. Further weight loss at higher temperature might be due to the loss of organic components inside the intercalated particle. The overall composition of PI was about $20 \mathrm{wt}$. $\%$. This is somewhat higher than the organic composition in seashell nacre. 

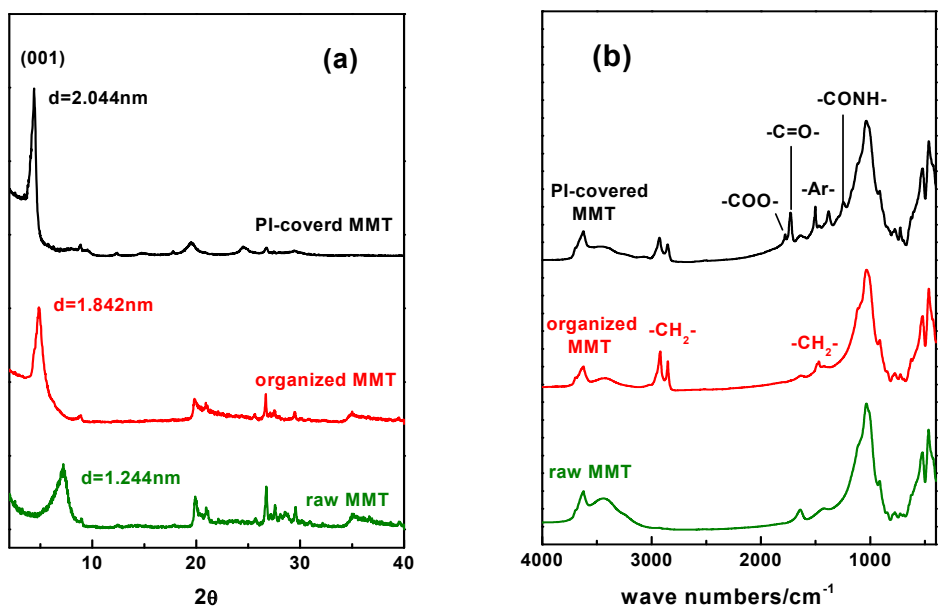

Fig. 2. XRD (a) and FTIR (b) analysis show the well combination of clay nano-platelets and polyimide

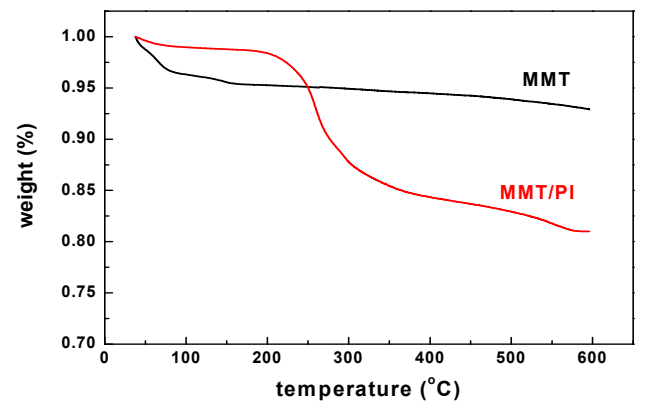

Fig. 3. Thermal gravimetric analysis shows the loss of organic content when artificial nacre heated to high temperature, and the percentage of PI is almost $20 \mathrm{wt} . \%$

Montmorillonite platelets covered by PI macromolecules then form the layered nanostructure by centrifugal deposition. In this process, clay platelets become preferentially parallel to the surface of substrate as the leaves fall and spread flatly on the ground due to gravity. SEM images of the cross-sectional view of the composite exhibit a high degree of ordering of PI/MMT multilayers (Fig. 4a). AFM shows that clay platelets are glued together with adjacent platelets by polyimide macromolecules (Fig. $4 \mathrm{~b}$ ). The deposition process and formation of nanostructure can be represented by the schematics in Fig. $4 \mathrm{c}$ and $\mathrm{d}$. The layered nanostructure is also confirmed by X-ray diffraction (Fig. 5a), the ratio of diffraction intensities of the centrifugally deposited sample is $\mathrm{I}(001) / \mathrm{I}(100)=62$ which is much higher than that of normally deposited sample with $\mathrm{I}(001) / \mathrm{I}(100)=12$. This confirms that the MMT platelets were preferentially aligned in parallel to the substrate in the centrifugal deposition 
process. Another advantage of this technique is that the deposition process is much faster so that thick films over $100 \mu \mathrm{m}$ can be easily obtained in a few minutes (Fig. 5b). The efficiency is much higher than other methods.
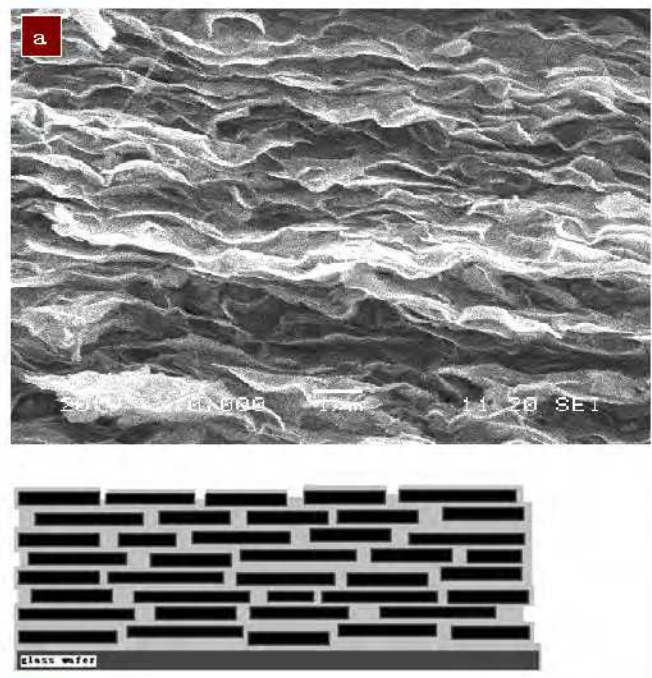

d
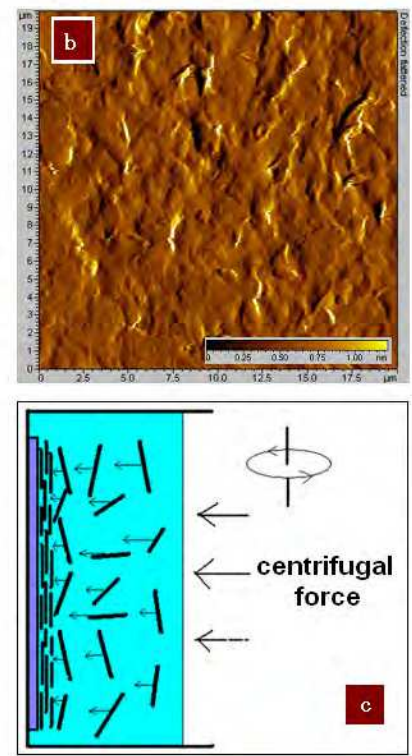

Fig. 4. The ordered nacre structure of the PI/MMT composite, a) clay platelets array in composite, b) organic platelets inset in polymer, c) the automatic alignment of clay platelets under the centrifugal force, $d$ ) representation of the general ordered nanostructure.

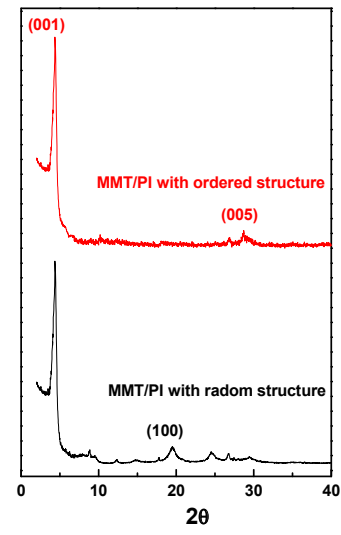

(a)

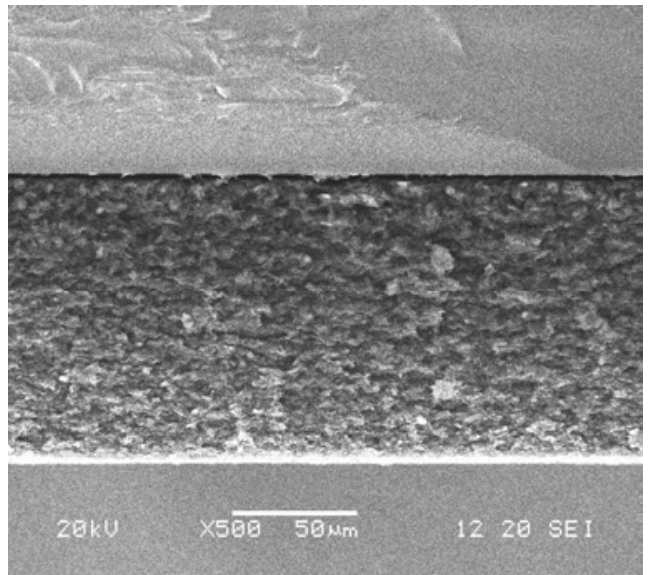

(b)

Fig. 5. Slice of PI/MMT composites acquired by the centrifugal deposition, (a) clay platelets become parallel to the substrate with the orientation of $(001)$, (b) the sample with ordered structure in nano-scale with the thickness over $100 \mu \mathrm{m}$. 
PI/MMT films were separated from the glass substrate using hydrofluoric acid so that the free-standing films were obtained and tested. The composites exhibited excellent and unique mechanical properties compared to PI polymer as shown in Fig. 6. Typical tensile stress-strain curves present a metal-like behaviour in which a plastic deformation stage occurs after initial elastic deformation. This is not observed in disordered composites and monolithic polyimide (PI). Under a stress below $45 \mathrm{MPa}$, both ordered and disordered artificial nacres exhibit an elastic characteristic. Beyond this stress, there is a platform stage for the ordered composite which is attributed to the debonding of organic-inorganic interface as described by Evans A.G (Evans et al., 2001). The following plastic deformation and breakage stages are rather similar to polyimide polymer (PI) for both ordered and disordered artificial nacres.

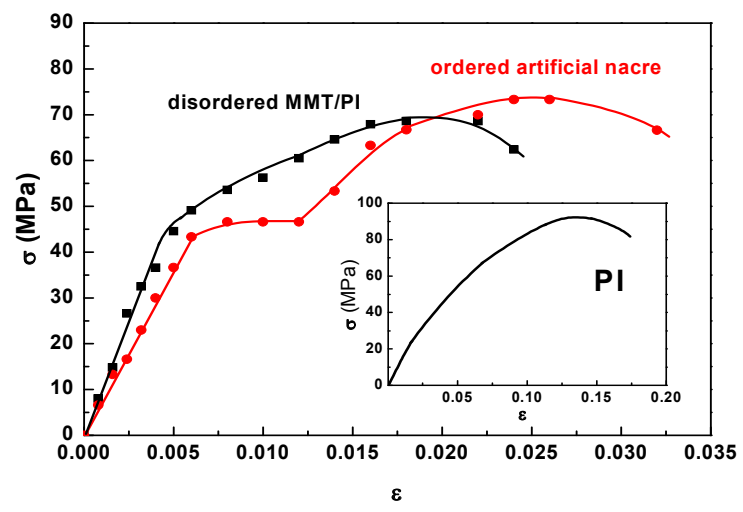

Fig. 6. In contrast to PI material and PI/MMT with disordered structure, ordered artificial nacre is yielding under a stress, which presents a unique metal-like behaviour.

The ordered nanocomposite exhibited an ultimate tensile stress $\left(\sigma_{\mathrm{u}}\right)$ of about $80 \mathrm{MPa}$ and a Young's modulus E of 7-9 GPa as summarized in Table 1. The strength is comparable to that of clay-polyelectrolyte composites with disordered structures (Tang et al., 2003) and seashell nacre (Evans et al., 2001). The Young's modulus is lower than that of abalone seashell nacre due to higher organic content in the PI/MMT composite. Nevertheless, the obtained E values are comparable to that of plywood bones with lamellar morphology and significantly higher than that of clay-polyelectrolyte composites reported (Tang et al., 2003). Nano-indentation was used to determine the hardness of PI/MMT composites. The results are in the range of 1.4-1.6 GPa, as shown in Table 1, which is almost 10 times higher than PI. It is noted that the PI/MMT composite with ordered laminated nanostructure has lower modulus and higher hardness than that with disordered structure. This is because the inorganic platelets in disordered structure interlock with each other and exert higher resistance to the deformation of the macromolecules than the ordered brick-and-mortar structure. On the other hand, the higher hardness of the ordered structure is attributed to the higher probability for the indenter to meet the hard MMT platelets when indenter is being pressed into the surface. As a result, the ordered structure exhibits similar mechanical properties to lamella bones. 


\begin{tabular}{|c|c|c|c|c|c|}
\hline Materials & $\begin{array}{c}\text { Young's } \\
\text { modulus E } \\
(\mathrm{GPa})\end{array}$ & $\begin{array}{c}\text { Strength } \\
\sigma_{\mathrm{u}}(\mathrm{MPa})\end{array}$ & $\begin{array}{c}\text { Extensibility } \\
(\%)\end{array}$ & $\begin{array}{c}\text { Hardness } \\
(\mathrm{GPa})\end{array}$ & Reference \\
\hline $\begin{array}{c}\text { PI/MMT- } \\
\text { ordered }\end{array}$ & $8 \pm 0.5$ & $80 \pm 5$ & $3.5 \pm 0.2$ & $1.5 \pm 0.1$ & Present \\
\hline $\begin{array}{c}\text { PI/MMT- } \\
\text { disordered }\end{array}$ & $9 \pm 1$ & $69 \pm 8$ & $2.5 \pm 0.2$ & $1.2 \pm 0.2$ & Present \\
\hline PI & $3 \pm 1$ & $90 \pm 10$ & $17 \pm 2$ & $0.2 \pm 0.05$ & Present \\
\hline $\begin{array}{c}\text { Seashell } \\
\text { nacres }\end{array}$ & $66-81$ & $194-248$ & 1.1 & - & $\begin{array}{c}\text { Wang et } \\
\text { al., } 2001\end{array}$ \\
\hline $\begin{array}{c}\text { Artificial } \\
\text { nacre }\end{array}$ & $9-13$ & $95-109$ & $7-10$ & - & $\begin{array}{c}\text { Tang et al., } \\
2003\end{array}$ \\
\hline $\begin{array}{c}\text { Cortical } \\
\text { bone }\end{array}$ & $7-30$ & $100-230$ & $1-3$ & - & $\begin{array}{c}\text { Bonfield et } \\
\text { al., } 1998\end{array}$ \\
\hline
\end{tabular}

Table 1. Mechanical properties of PI/MMT composites

(The data was obtained at a relative humidity of $38 \%$ and $\mathrm{T}=25^{\circ} \mathrm{C}$.)

\subsection{Summary}

In summary, the inorganic-organic (PI/MMT) composites with ordered structure in nanoscale have been successfully prepared by a simple centrifugal deposition process. The whole process is the one that simulates and accelerates the natural process of nacre growth, including the formation of organic and inorganic components, adsorption of organic compound to inorganic frameworks, and matter deposition. The structural similarity between the PI/MMT composite and natural nacre gives a similarity of properties, such as mechanical strength, which is attributed to strong interfacial interaction and high compatibility of the components. Structural and functional resemblance makes clay-polymer multilayer composite a close replica of natural biomaterials. This approach is also applicable to a wide range of artificial nacre materials with different organic and inorganic building blocks.

\section{Electrophoretic deposition process and in-situ polymerization to fabricate polyacrylamide/montmorillonite (PAM/MMT) nacre-like nanocomposite}

Electrophoretic deposition (EPD) is a rapid and low cost process, and capable of producing materials with complex geometry. An EPD process consists of two steps. In the first step, particles suspended in a liquid medium are driven towards an electrode by applying an electric field to the suspension (electrophoresis). In the second step, the particles are collected at one of the electrode and form a coherent deposit on it (deposition).

In this section, the fabrication of polyacrylamide/montmorillonite (PAM/MMT) nanocomposites with nacre-like structure is presented as a case study of electrophoretic deposition and in-situ polymerization process. Montmorillonite was first modified by acrylamide, and suspended in distilled water. After electrophoretic deposition, the film was polymerized by ultraviolet-radiation. The ordered "brick-and-mortar" structure was made 
of montmorillonite and polyacrylamide. The composites exhibited outstanding properties that approached those of nacre.

\subsection{Experimental procedure}

\subsubsection{Materials}

$\mathrm{Na}^{+}$type montmorillonite (Na-MMT) with a cation exchange capacity of 90 meq / (100 g) was provided by Zhejiang Fenghong Clay Chemicals Co. Ltd. Acrylamide was used as an intercalating agent and reacting monomer, and benzyl was used as a free-radical photon initiator. Both were used as-received without further purification. The light source was a 150 $\mathrm{W}$ photochemical reactor lamp (Traper, Japan). Sodium polymeta-phosphate (SPP), tetrachloromethane and methanol were chemically purified.

\subsubsection{Preparation of organic clay}

To make the organophilic clay (O-MMT), $10 \mathrm{~g}$ of Na-MMT was dispersed in $300 \mathrm{ml}$ deionized water with vigorous stirring for 3 hours. Meanwhile an acrylamide solution contained $1 \mathrm{~g}$ of acrylamide in $60 \mathrm{ml}$ of deionized water was prepared by the addition of 10 wt $\% \mathrm{HCl}$ to adjust the $\mathrm{pH}$ value to about 3 4. Then the protonated acrylamide solution was added slowly to the MMT suspension. The hydrophobic clay was recovered by centrifugation and filtration of the solution, followed by repeated rinse to remove excess ammonium ions. Finally, the clay was dried at $80^{\circ} \mathrm{C}$ in a vacuum oven overnight and then grinded and sieved to 200 mesh.

\subsubsection{Electrophoretic deposition}

The electrophoretic deposition of O-MMT onto the electrode surface was carried out in a 150 $\mathrm{ml}$ beaker containing $100 \mathrm{ml}$ O-MMT $(0.01 \mathrm{~g} / \mathrm{ml})$ aqueous solution with addition of SPP $(0.001 \mathrm{~g} / \mathrm{ml})$ as a surfactant. Before the deposition, the solution was vigorously stirred for 3 hours and aged overnight to get a stable suspension. Two pre-cleaned stainless steel plates with a surface area of $4 \mathrm{~cm}^{2}$ served as both the working electrode and the counter electrode. The electrophoretic deposition of O-MMT solution was performed following a potentiostatic procedure with a working voltage of $5 \mathrm{~V}$. The distance between the two electrodes was $2 \mathrm{~cm}$. And this procedure lasted for 5 minutes to get a uniform off-white coating. The coated electrodes were immediately withdrawn from the emulsion after electrophoretic deposition and rinsed with tetrachloromethane to remove the residue of the acrylamide monomers.

\subsubsection{Photo polymerization of PAM}

In a typical procedure, $7.5 \mathrm{~g}(0.075 \mathrm{~mol})$ of benzyl was dissolved in $150 \mathrm{ml}$ of methanol solvent under vigorous magnetically stirring for 3 hours. Then the coated electrodes were put into the solution and irradiated with a UV light for 24 hours (Fig. 7). After drying in a vacuum oven at $60{ }^{\circ} \mathrm{C}$ for 24 hours, the off-white films were peeled off from the electrodes by a sharp blade for microstructure analysis and mechanical testing. 


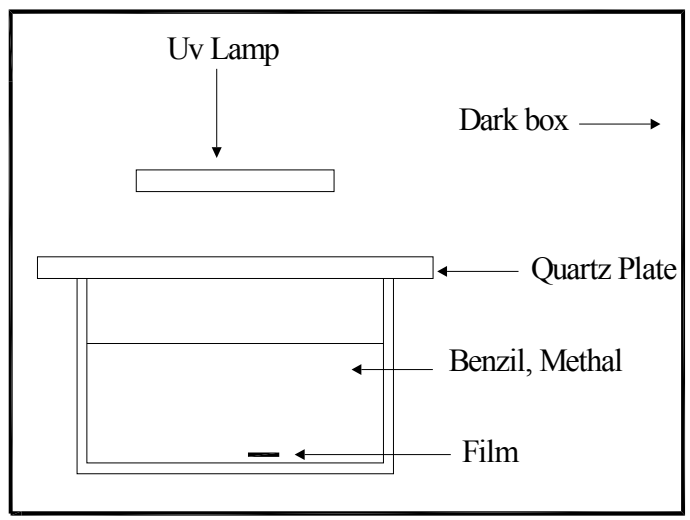

Fig. 7. Experimental setup of photo polymerization for the preparation of PAM/MMT

\subsubsection{Apparatus}

The montmorillonite platelets and microstructure of PAM/MMT composites were examined using field emission scanning electron microscopy (JEOL, JSM-6400, Japan) with an estimated resolution of $\sim 5 \mathrm{~nm}$. X-ray diffraction was taken on D8-Discover (Germany) to examine the highly ordered laminated structure, and FTIR spectrum (SYSTEM 2000, USA) was used to determine the organic compounds in this organic-inorganic composites. Nanoindentation (Berkovich tip, radius of curvature $50 \mathrm{~nm}$ ) was taken to determine the mechanical properties. TGA (SETARAM TGA92, France) was used to determine the formation and content of organic compounds in the composites.

\subsection{Results and discussion}

\subsubsection{Effect of monomer and polymerization on d-spacing of Na-MMT's (001) diffraction peak}

The replacement of inorganic cations on the surfaces by quarternary ammonium ions expands the clay galleries. Fig. 8 shows the XRD patterns of the various MMT films obtained. The Na-MMT film was also prepared by electrophoretic deposition in the NaMMT suspension. The d-spacing of Na-MMT's (001) diffraction peak was $1.29 \mathrm{~nm}$, which was increased to $1.89 \mathrm{~nm}$ when modified by acrylamide monomers. It is implied that the monomer was intercalated into the interlayer of the clay. But when the monomers were polymerized, the d-spacing decreased to $1.35 \mathrm{~nm}$. This is because the polymerization of AM to PAM gave a contraction of the layers. The polymer between the layers of the clay might have adopted a flattened conformation (Lan et al., 1994), whereas the monomers intercalated into the clays adopted random conformations. We can also find preferred orientation of the composites by the changes of the diffraction peaks. When the clay was intercalated by the monomers, the (001) diffraction peak became very strong. After the monomers were polymerized, it became weaker while other peaks even disappeared. This indicated that the composites exhibited a higher degree of ordering in the PAM/MMT multilayer after polymerization. 


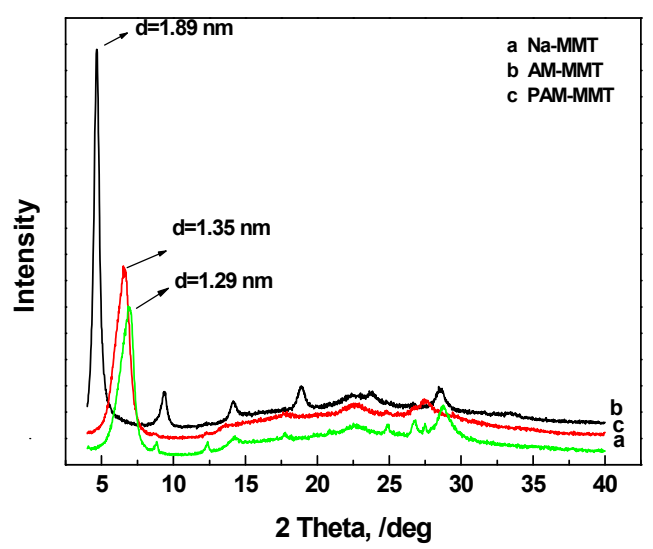

Fig. 8. XRD patterns of various MMT films: (a) Na-MMT; (b) AM-MMT and (c) PAM-MMT.

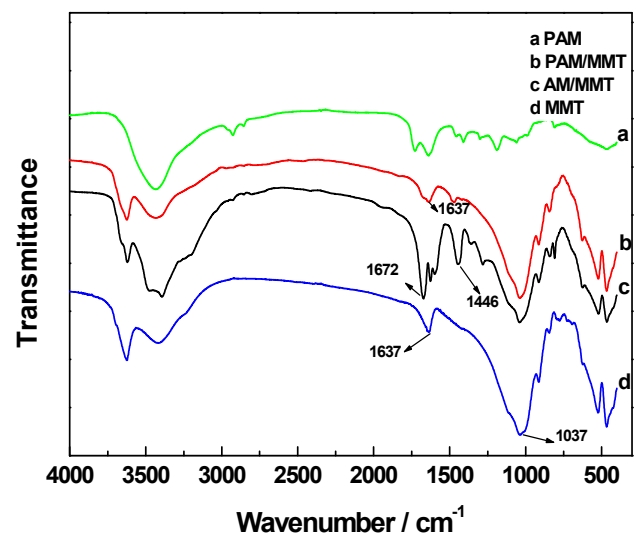

Fig. 9. FTIR spectra of the PAM (a), PAM-MMT (b), AM-MMT (c) and Na-MMT (d)

\subsubsection{FTIR analysis}

The characteristic FTIR spectra of the Na-MMT, AM-MMT, PAM-MMT and PAM are given in Fig. 9. The representative absorption peaks at $1037 \mathrm{~cm}^{-1}$ and $1637 \mathrm{~cm}^{-1}$ contributed to $\mathrm{OH}$ group on montmorillonite. The absorption peak at $1672 \mathrm{~cm}^{-1}$ was attributed to $-\mathrm{CONH}_{2}$ on acrylamide. And the characteristic peak at $1446 \mathrm{~cm}^{-1}$ was attributed to $-\mathrm{CH}_{2}-$. The absorption peaks of $-\mathrm{CONH}_{2}$ and $-\mathrm{CH}_{2}$ - became smaller when polymerized under the UV light because the $\mathrm{OH}$ group on the surface of montmorillonite participated in the reaction. Hence, the absorption peak at $1637 \mathrm{~cm}^{-1}$ contributed to $\mathrm{OH}$ group on PAM/MMT film became weaker compared to that of MMT. 


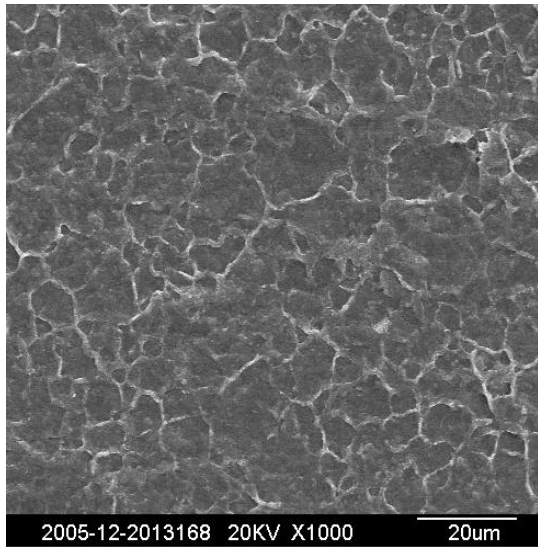

(a)

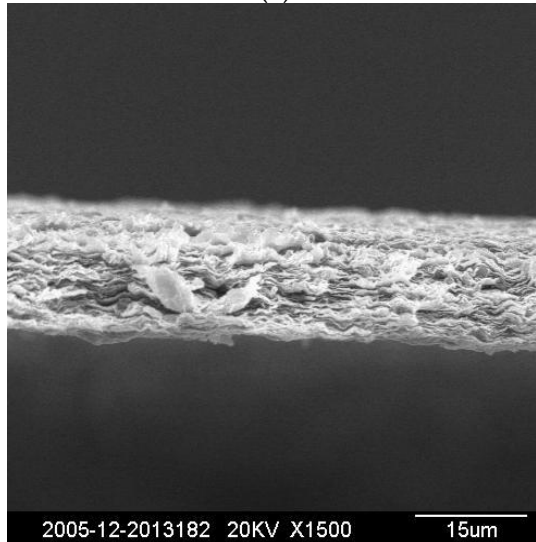

(c)

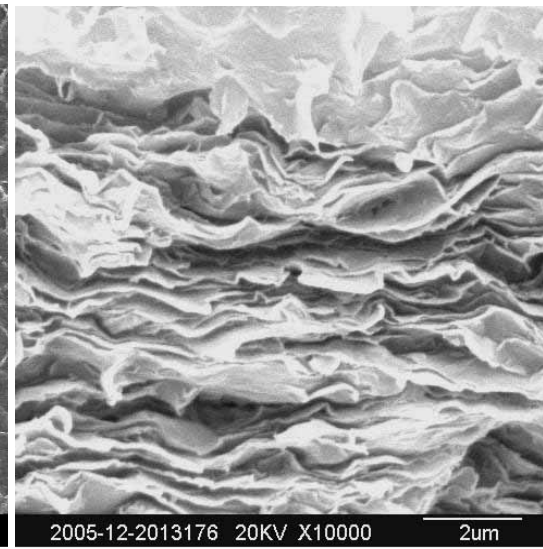

(b)

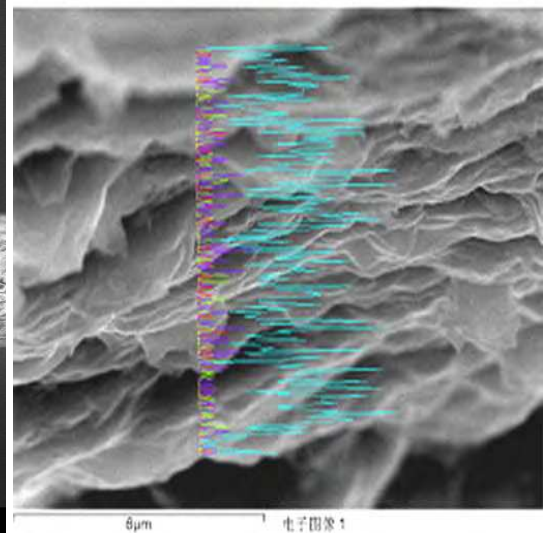

(d)

Fig. 10. SEM micrograph for electrophoretic deposited PAM/MMT film: (a) the surface of the film; (b) the cross-sectional film at a magnification of $10,000 \times$; (c) the cross-sectional film at a magnification of 1500x; (d) SEM image of a cross-sectional PAM/MMT film with a EDS line scan analysis of $\mathrm{Al}$ (blue) , C (red) ,O (yellow ) and Fe (purple) elements.

\subsubsection{Microstructure of layered nanocomposites}

The SEM images of the polymerized film are shown in Fig. 10. It can be seen that the polymerized film exhibits layered structure similar to the structure of nacre. The formation process of the film can be described as follows. First, the monomer is intercalated into the interlayer of MMT, which was stated before. Second, the electrophoretic deposition of MMT platelets is carried out. Montmorillonite is a clay mineral consisting of stacked silicate sheets approximately $1 \mathrm{~nm}$ thick and approximately $200 \mathrm{~nm}$ long. Its grains possess a high aspect ratio and demonstrate platelet morphology. As we know, the montmorillonite platelet presents negative charges on the large basal faces upon hydration. By contrast, the sign and density of the charges at the edges of the platelets are $\mathrm{pH}$ dependent, positive edges being found in acidic media (Graham et al., 1995). However, the overall particle charge is 
invariably negative. In our experiment, we adjusted the $\mathrm{pH}$ of the emulsion to 10 to avoid the edge effect, which may affect the arrangement of the clay platelets. As we expected, the film was uniformly deposited onto the anode. From the cross section of the film (Fig. 10b and c), we obtained an orderly layered structure, which was similar to the "brick-andmortar" structure of the nacre. The clay platelet was about decades of nanometers in thickness, and several micrometers in width. They were stacked tightly on the direction parallel to the surface of the electrodes. From Fig. 10a, we can see a uniform and smooth surface of the thin film with some overlapping clay platelets that exhibit white networking curves. The smoothness of the surface is attributed to the unique property of a high aspect ratio of the clay particle. The two-dimensional sheets can cover the defects of the underlying layers. This phenomenon was called "self-healing" by Kleinfeld and Ferguson (Kleinfeld \& Ferguson, 1994). In Fig. 10d, an energy-dispersive X-ray detector attached to the SEM was used for evaluation of the $\mathrm{Al}, \mathrm{Fe}, \mathrm{O}$ and $\mathrm{C}$ distribution in the cross-section of the $\mathrm{PAM} / \mathrm{MMT}$ film. $\mathrm{Al}, \mathrm{Fe}$ and $\mathrm{O}$ are from MMT, and $\mathrm{C}$ is from PAM. The curves show an alternating distribution of the $\mathrm{Al}$ and $\mathrm{C}$ elements, which indicates the alternating organicinorganic layered structure. From Fig. 8, we know that the interlayer of the film was filled with networking polymer which could bind clay platelets together since the d-spacing of (001) peak was enlarged by it. And in Fig. 11, the TGA curves show that the polymer content is about $4.7 \mathrm{wt} \%$, i.e., the inorganic content was about $95.3 \mathrm{wt} \%$. Therefore, we can draw a conclusion that the polyacrylamide-clay nacre-like nanocomposites prepared in the present work consists of ordered montmorillonite layers and the networked polyacrylamide which was located between the montmorillonite layers.

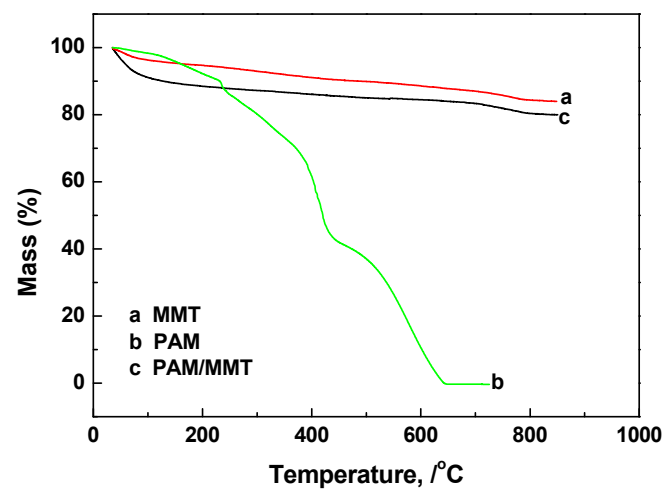

Fig. 11. TGA curves of MMT (a), PAM (b), and PAM/MMT (c).

\subsubsection{Mechanical properties of the composites}

Nano-indentation was used to determine mechanical properties of these samples with ordered nanostructure. With the laminated structure similar to nacre, the films demonstrated outstanding properties. Table 2 shows the typical modulus values of the ordered PAM/MMT composites acquired by nano-indentation. According to Table 2, the Young's modulus of the PAM/MMT composites is about $16.92 \mathrm{GPa}$. The high Young's modulus is attributed to a relatively high inorganic content $(95.3 \%)$ and excellent 
organic/inorganic bonding in the PAM/MMT composite. Nevertheless, the obtained E value was close to or even exceed the Young's modulus of plywood bones with lamellar morphology, and significantly exceed the Young's modulus of hybrid clay-polyelectrolyte composites with disordered structures reported by Tang et al, 2003. Table 2 also shows the typical hardness values of PAM/MMT composites acquired by nano-indentation. The hardness was about $0.95 \mathrm{GPa}$. For comparison, we prepared the pure montmorillonite film without polymer-intercalation under the same conditions which has a modulus of $2.92 \mathrm{GPa}$ and a hardness of $0.20 \mathrm{GPa}$. It can be seen that the ordered laminated film exhibited higher strength and rigidity. This is because the brick-and-mortar structure can be stretched consistently during the elastic deformation, which is a way to absorb energy. Inorganic platelets can also interlock with each other and exert high resistance to the deformation of the macromolecules.

\begin{tabular}{|c|c|c|}
\hline Materials & Young's modulus E (GPa) & Hardness (GPa) \\
\hline $\begin{array}{c}\text { Monolithic } \\
\text { MMT }\end{array}$ & 2.92 & 0.20 \\
\hline PAM/MMT & 16.92 & 0.95 \\
\hline
\end{tabular}

Table 2. Mechanical properties of pure MMT and PAM/MMT composites.

\subsection{Summary}

Polyacrylamide-clay nanocomposites were successfully prepared by electrophoretic deposition of montmorillonite aqueous suspension modified by acrylamide monomers and polymerized by ultraviolet-radiation. Acrylamide monomers functioned as both the intercalating agent and reacting monomers. The results show that the nanocomposites possess a uniform and ordered layer-by-layer structure with montmorillonite sheets as inorganic layers and polyacrylamide as interlayer phase, which is similar to the "brick-andmortar" structure of the natural nacre. The nacre-like composites have outstanding mechanical properties, hardness and Young's modulus are $0.95 \mathrm{GPa}$ and $16.92 \mathrm{GPa}$, respectively, which are close to or even exceed the Young's modulus of plywood bones with lamellar morphology with $\mathrm{E}=6 \sim 16 \mathrm{GPa}$.

\section{Special assembly of laminated acrylic anodic electrophoretic resin / montmorillonite (AAER/MMT) nanocomposite that mimics nacre}

In this section, a special assembly method, hydrothermal-electrophoretic assembly, is proposed to prepare acrylic anodic electrophoretic resin/montmorillonite (AAER/MMT) nanocomposite that mimics nacre, both in structure and composition. The two-step assembly process includes intercalation of polymer into interlayer space of montmorillonite by hydrothermal process and the subsequent electrophoretic deposition.

$\mathrm{Na}^{+}$-montmorillonite (NMMT) platelets possess high aspect ratio (the silicate sheet thickness is $<1 \mathrm{~nm}$, and the lateral dimensions may vary from $30 \mathrm{~nm}$ to several microns or larger), with negative charges on their basal faces. It is this special character that inspired us in introducing electrophoretic deposition (EPD) into the assembly of montmorillonite platelets to form ordered nanolaminated composite films. On the other hand, acrylic anodic 
electrophoretic resin (AAER) was chosen as the organic phase in the section, which has been widely used both in industry (e.g. almost all mass-produced steel car bodies) and scientific research due to its well-known electrophoretic ability, film forming ability, adhesive strength with various surfaces, corrosion resistance and insulating property.

In order to assemble bricks and mortar in nano-scale, we borrowed the method of intercalation from traditional hybrid preparation. A series of significant work done by $\mathrm{Li}$ and his colleagues ( $\mathrm{Li}$ et al., 2002; Chen et al., 2002; Wang \& Li, 2002) on preparing laminated structures gave us inspiration in polymer intercalation to obtain the hydrothermally-intercalated montmorillonite (HMMT) powder, under the force of the hydrothermal process.

It is hoped that the hydrothermal-electrophoretic assembly process (Scheme as shown in Fig.12) would open fascinating possibilities for assembling a variety of laminated nanocomposites.
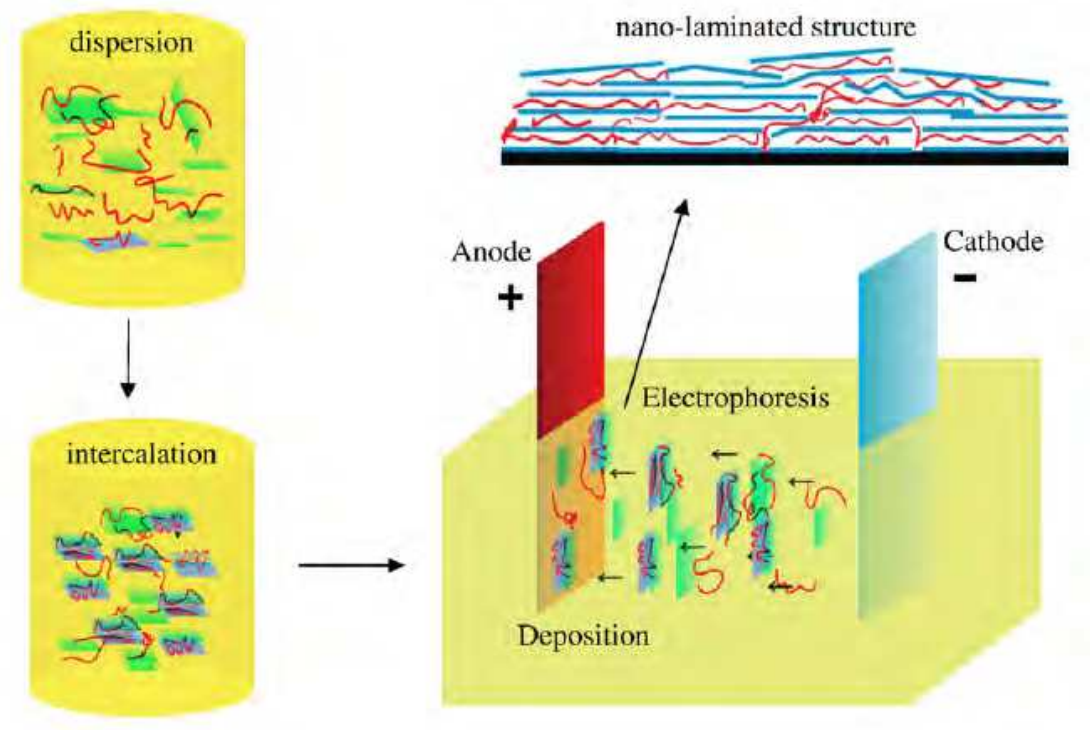

Fig. 12. The schematic showing the hydrothermal-electrophoretic assembly to construct nanolaminated film structure.

\subsection{Experimental methods and materials}

\subsubsection{Materials}

NMMT was donated by Zhejiang Fenghong Clay Chemicals Co., Ltd., with a cation exchange capacity (CEC) of $90 \mathrm{meq} /(100 \mathrm{~g})$ and average size of $\sim 1 \mu \mathrm{m}$. AAER (Commodity No. DT323-75) synthesized from methacrylic acid, methyl methacrylate, butyl methacrylate, 2-hydroxyethyl methacrylate, styrene and glycidyl methacrylate and neutralized by triethylamine, was supplied by Tianjing Dengta Co., Ltd. The acidic value of AAER before neutralization is $\sim 105 \mathrm{mgKOH} / \mathrm{g}$ (the value is provided by the supplier). The weight- 
average molar mass and poly-dispersity index for the AAER used are 20,290 g/mol and 2.99 , respectively, determined by gel permeation chromatography using Viscotek TriSEC Model 302. No inorganic or organic filler was present in the original AAER. Bisphenyl A type epoxy resin (E-44) was obtained from Wuxi Lanxing Co., Ltd. Cyclohexanone, dimethyl acetamide and sodium hydroxide were purchased from Beijing Chemical Reagents Company and used without further purification. Ultrasonic machine was JY92-П (Ningbo Scientz Biotechnology Co., Ltd.).

\subsubsection{Preparation of aqueous suspension for subsequent EPD}

NMMT suspension was prepared as follows: $1.0 \mathrm{~g}$ of NMMT was dispersed in $100 \mathrm{ml}$ o deionized water, stirring for 24 hours. Then the suspension was adjusted to $\mathrm{pH}=\sim 8.5$ using 1 wt.\% aqueous solution of sodium hydroxide, followed by ultrasonic dispersion (500 W, 20 min). Hydrothermal method was employed to prepare HMMT suspension. $0.1 \mathrm{~g}$ of AAER was added into $100 \mathrm{ml}$ of NMMT suspension before being transferred into a Teflonlined autoclave with a stainless steel shell. The autoclave was kept at $130{ }^{\circ} \mathrm{C}$ for 24 hours and then allowed to cool to room temperature naturally. The resulting aqueous suspension was further dispersed by ultrasonic homogenizer ( $500 \mathrm{~W}, 20 \mathrm{~min}$ ) for subsequent EPD directly.

\subsubsection{Electrophoretic deposition (EPD)}

Constant voltage was applied during the EPD process using a battery testing system (CT3008B-5V20mAS1, NEWARE, China). The electrodes used were 18Cr8Ni type stainless steel substrates for both anode and cathode, with effective area of approximately $3 \mathrm{~cm}^{2}$. Electrodes were polished before use. EPD under $2.5 \mathrm{~V}$ for $15 \mathrm{~min}$ was adopted for all the films studied in this article, with the electrode spacing of $1.0 \mathrm{~cm}$. The as-deposited films (on substrate) were carefully rinsed with de-ionized water and then dried in the air.

\subsubsection{Heat-treatment of films}

In a typical curing process, after drying in the air, the stainless steel substrates carrying films were put into a baking oven and left at $170{ }^{\circ} \mathrm{C}$ for 1 hour to cure.

\subsubsection{Preparation of powders}

HMMT powder was collected by filtration from the HMMT suspension and washed with hot de-ionized water several times till ultraviolet absorption spectrum indicated the absence of $\mathrm{C}=\mathrm{O}$ in water. The obtained powder was dried at $50{ }^{\circ} \mathrm{C}$ in the air for 24 hours. In an attempt to demonstrate that hydrothermal (solvent-thermal) method is universally feasible in intercalating oligomer molecules into the basal layer space of NMMT platelets, similar solvent-thermal process was also applied to intercalate E-44 epoxy resin molecules into NMMT platelets using dimethyl acetamide as the solvent; the powder obtained was designated as SMMT. For comparison, with the help of FTIR, the AAER molecules that had been intercalated into clay interlayer space and those that had been mixed physically with clay platelets without intercalation, we prepared mixture powder of AAER and NMMT by centrifugal sedimentation (from suspension containing $0.1 \mathrm{~g}$ of AAER and $1 \mathrm{~g}$ of dispersed NMMT powder). 


\subsubsection{Characterization}

XRD experiments were carried out in D/max-rB X-ray diffractometer, using $\mathrm{Cu} \mathrm{K} \alpha$ radiation $(\lambda=1.5418 \AA)$. Care was taken to minimize the off-axis error during sample preparation. Parameters for the continuous scan mode are $0.01^{\circ}, 3^{\circ} / \mathrm{min}$.

Surface and cross-section observations and thickness measurement of the films were carried out by scanning electron microscope (SEM) in a JEOL JSM-6400 with an operating voltage at $10 \mathrm{kV}$. Energy Dispersive Spectroscopy (EDS) with a resolution of $\sim 1 \mu \mathrm{m}$ was collected at 20 $\mathrm{kV}$, counting for $10 \mathrm{~min}$. For thickness measurement, the film was peeled off the stainless steel substrate with special care and spread on the glass plate using water-soluble glue.

FTIR experiments were performed at ambient temperature with a spectrometer (Spectrum GX, PerkinElmer, USA) at a resolution of $\sim 4 \mathrm{~cm}^{-1}$. Care was taken when pressing $\mathrm{KBr}$ pellets to minimize effect of pressure on peak frequencies. 5 spectra were taken of each specimen and the average position of each peak (located automatically by peak picking software attached to the testing system) was then determined, with reproducibility of $<1 \mathrm{~cm}^{-1}$.

Nano-indentation (XP CSM, XP-B1819n tip) was used to test elastic moduli of NMMT and HMMT films. At least 15 indentations were conducted on each sample surface. The mean and standard deviation of the reduced Young's modulus of a sample was calculated from the indentations by rejecting 2 of the highest and 2 of the lowest values to remove the outliners in a consistent manner.

The weight percentage of the organic component contained in films was determined by thermal gravimetric analysis on a TGA2050 gravimetric analyzer, and samples were heated in the air from ambient temperature to $700{ }^{\circ} \mathrm{C}$ at a rate of $20^{\circ} \mathrm{C} / \mathrm{min}$.

Conductivity meter (DDS-12D, Lida, China) and Zeta Potential Analyzer (Zeta Plus, Brookhaven, USA) were employed to study the electrical conductivity and stability of powder in suspensions under ambient temperature, respectively.

\subsection{Results and discussion}

\subsubsection{Effectiveness of hydrothermal method}

Fig. 13a shows a series of XRD patterns corresponding to NMMT powder (A), HMMT powder (B), SMMT powder (C). After hydrothermal intercalation, an expansion in (001) layer space (designated as $\mathrm{d}_{001}$ ) is observed, from $1.29 \mathrm{~nm}$ of NMMT powder (trace $\mathrm{A}$ ) to $1.51 \mathrm{~nm}$ of HMMT powder (trace B), similar to the results reported in literatures on hybrid preparation. Trace C indicates that intercalating E-44 molecules into NMMT platelets by similar solvent-thermal method was effective too, with associated $\mathrm{d}_{001}$ expanded to $1.60 \mathrm{~nm}$. Hereby, we may conclude that under appropriate conditions, hydrothermal (solventthermal) method is universally feasible in intercalating polymer/oligomer molecules into the basal layer space of NMMT. 

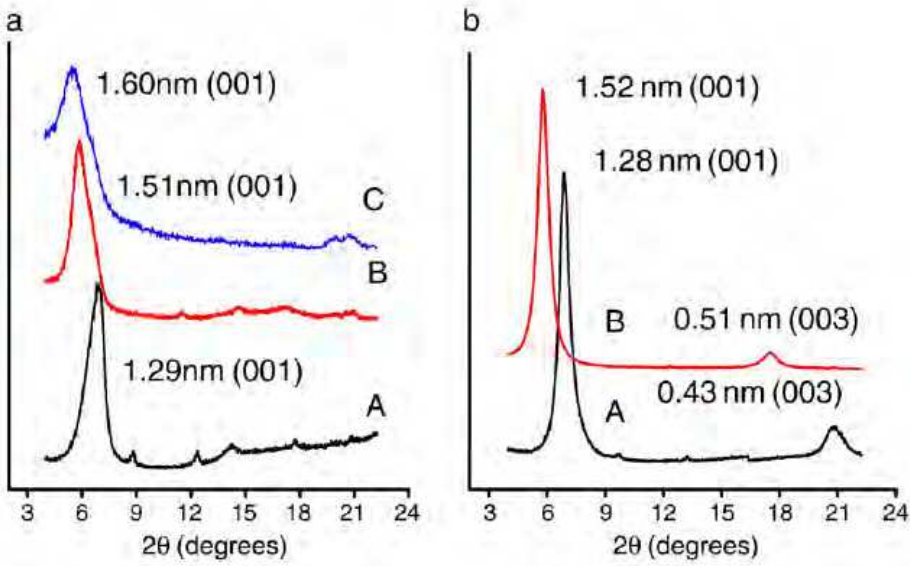

Fig. 13. a: XRD patterns of NMMT powder (A), HMMT powder (B) and SMMT powder (C). b: XRD patterns of NMMT film (A) and HMMT film (B).

Further evidence of the effectiveness of intercalation is found from FTIR, as shown in Fig. 14. In panel a, several transmittance bands and peaks typical of AAER film (trace A), which are absent in NMMT (trace D), appear in HMMT powder (trace B), including the methylene modes of $2950-2850 \mathrm{~cm}^{-1}$ and $1500-1400 \mathrm{~cm}^{-1}$ (arising from $\mathrm{CH}_{2}$ stretching and $\mathrm{CH}_{2}-$ scissoring vibration, respectively), and the $\mathrm{C}=\mathrm{O}$ stretching at $\sim 1730 \mathrm{~cm}^{-1}$. Two features are observed that provide evidence of the existence of polymer molecules confined within the interlayer space in HMMT. (1) As is shown in panel b, $v_{\text {as }}\left(\mathrm{CH}_{2}\right)$ and $v_{\mathrm{s}}\left(\mathrm{CH}_{2}\right)$ shift from 2934 $\mathrm{cm}^{-1}$ and $2877 \mathrm{~cm}^{-1}$ respectively in AAER to $2925 \mathrm{~cm}^{-1}$ and $2854 \mathrm{~cm}^{-1}$ respectively in HMMT powder. Panel c shows the frequency shift of $\delta\left(\mathrm{CH}_{2}\right)$ from $1453 \mathrm{~cm}^{-1}$ in AAER to $1463 \mathrm{~cm}^{-1}$ in HMMT powder. According to existing FTIR research on the conformation of aliphatic chains, $v_{\text {as }}\left(\mathrm{CH}_{2}\right)$ and $\delta\left(\mathrm{CH}_{2}\right)$ are sensitive to gauche/trans conformer ratio, their frequency shifts reflecting preferable conformation of methylene chains in specific environment. In general, freer chains tend to assume more gauche conformers than less-free chains, the latter tending to assume more trans conformers. Increasing conformer ratio of gauche to trans usually leads to frequency shift of $v_{\text {as }}\left(\mathrm{CH}_{2}\right)$ to lower value and of $\delta\left(\mathrm{CH}_{2}\right)$ to higher value. In our study, the frequency shifts of $v_{\mathrm{as}}\left(\mathrm{CH}_{2}\right)$ and $\delta\left(\mathrm{CH}_{2}\right)$ indicate that, in HMMT powder, AAER molecules tend to assume more gauche conformers, demonstrating that AAER molecules are confined (or at least partly confined) in clay layers so that interaction between AAER molecules are restricted. As for the $v_{\text {as }}\left(\mathrm{CH}_{2}\right)$ shift, although to the author's knowledge, no definite reason is yet known, it is supposed to be closely related to the confined statement of aliphatic chains. (2) Also in panel c, peaks at $1033 \mathrm{~cm}^{-1}$ ( $\mathrm{Si}-\mathrm{O}$ in-plane vibration) that are characteristic of NMMT, shift to $1016 \mathrm{~cm}^{-1}$ indicating that intercalated AAER molecules most probably interact with the silicate. In contrast, all these features do not appear in the mixture powder of AAER and NMMT (trace C). 


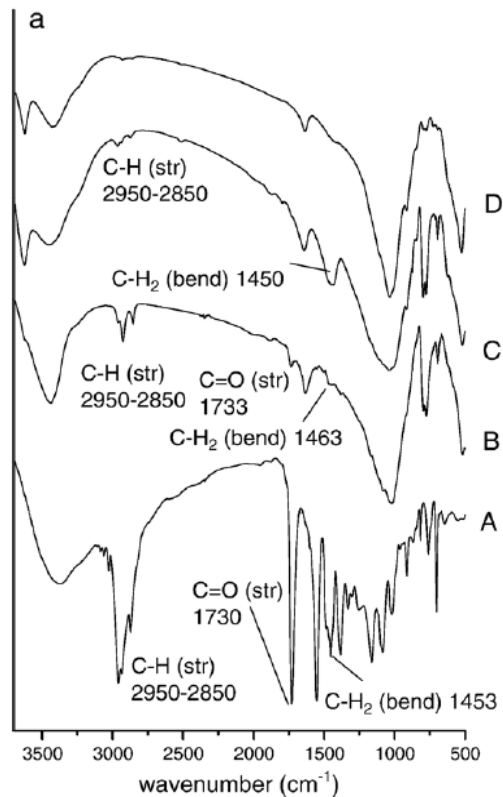

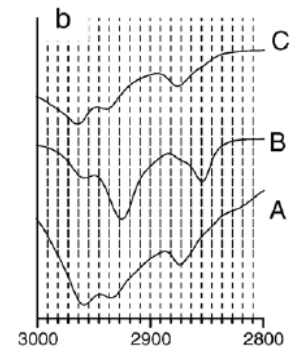

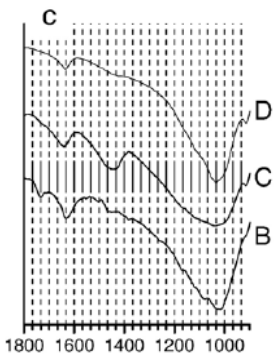

Fig. 14. a: FTIR transmittance spectra of AAER (A), HMMT powder (B), mixture powder of AAER and NMMT (C) and NMMT film (D). b and c: Enlargement of selected regions in panel a.

In thermodynamics, for the overall process, in which oligomer or polymer molecules are exchanged with the previously intercalated solvent molecules in the interlayer space, a negative variation in the Gibbs free energy is required. The driving force for the intercalation from solution is supposed to be the entropy gained by desorption of solvent molecules, which compensates for the decreased conformational entropy of the confined intercalated oligomer or polymer chains. Therefore, the variation in entropy in the overall process is positive, which means that high temperature in hydrothermal (solvent-thermal) process is beneficial in thermodynamics.

\subsubsection{Morphologies and structures of films}

The XRD pattern of the as-deposited HMMT film (trace B in panel b, Fig.13) displays a texture-like highly (00L) orientated structure with all other peaks generated by crystal planes besides (00L) nearly disappeared. Its $\mathrm{d}_{001}$ spacing was expanded to $1.52 \mathrm{~nm}$ for the asdeposited HMMT film, approximately consistent with that of HMMT powder (trace B in panel a, Fig.13), which means that the interlayer space is $\sim 0.52 \mathrm{~nm}$. In our study, no distinct variation in $\mathrm{d}_{001}$ was observed after heat treatment or after soaking in cyclohexanone. This is probably due to the hindrance against cross-linking for polymer molecules in the confined space. The orderly laminated structure of the HMMT film with a thickness of around $10 \mu \mathrm{m}$ can be observed from its cross-section SEM images (Fig.15a, b, c). By adjusting the voltage $(2 \sim 3 \mathrm{~V})$, electrode spacing $(1.0 \sim 2.0 \mathrm{~cm})$ and deposition time $(1 \sim 20 \mathrm{~min})$, the thickness of films (from hundreds of nanometers to $20 \mu \mathrm{m}$ ) could be controlled. In the EDS result of a 
line-scan (Fig.15c) on the cross-section of the HMMT film, carbon element was detected, even after the film was spoiled by ultrasonic processing in cyclohexanone, followed by washing several times with de-ionized water (Fig.15d). This once again demonstrates that polymer molecules exist in the interlayer space of the laminated composite films. But it is noted that not all platelets were intercalated with polymer, because XRD is an averaging technique and EDS gives statistical result concerning specific areas.
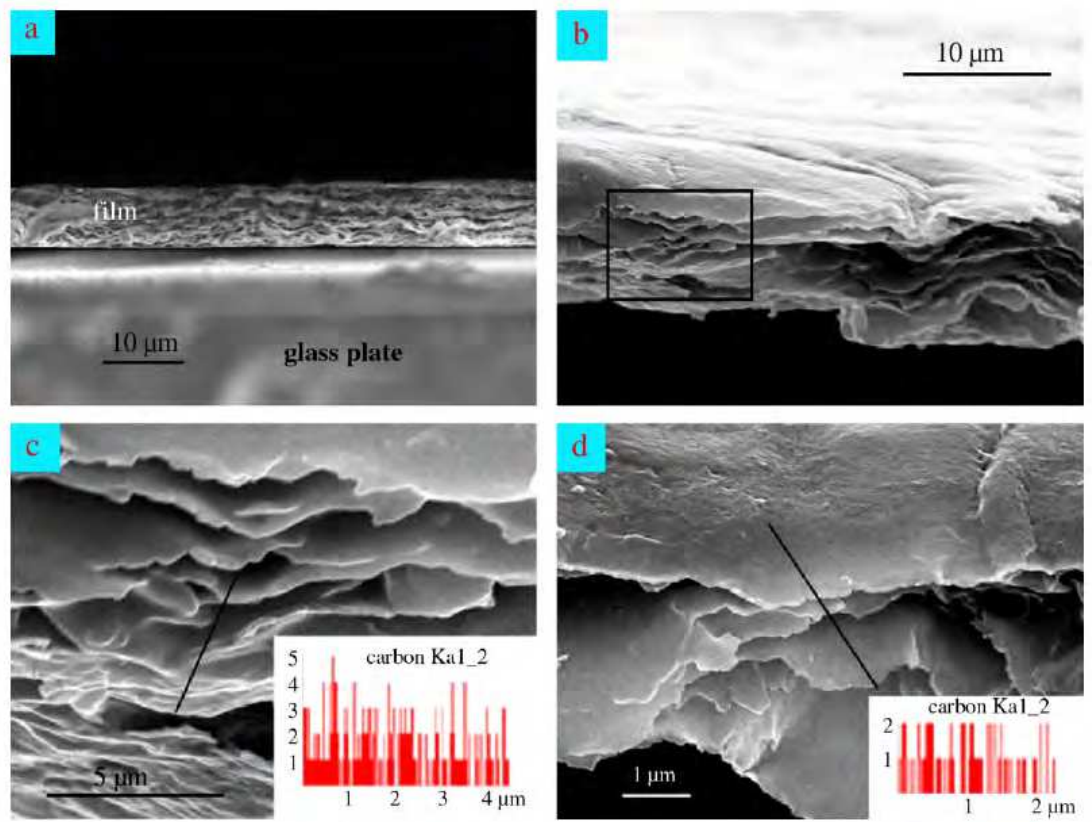

Fig. 15. SEM images of cross-section. a, b: the as-deposited HMMT film. c: enlargement of the box mark in image $b$ (inset: EDS line-scan result showing carbon element distribution along the dark line). d: the film after being spoiled by ultrasonic processing in cyclohexanone, followed by washing several times with de-ionized water (inset: EDS linescan result showing carbon element distribution along the dark line).
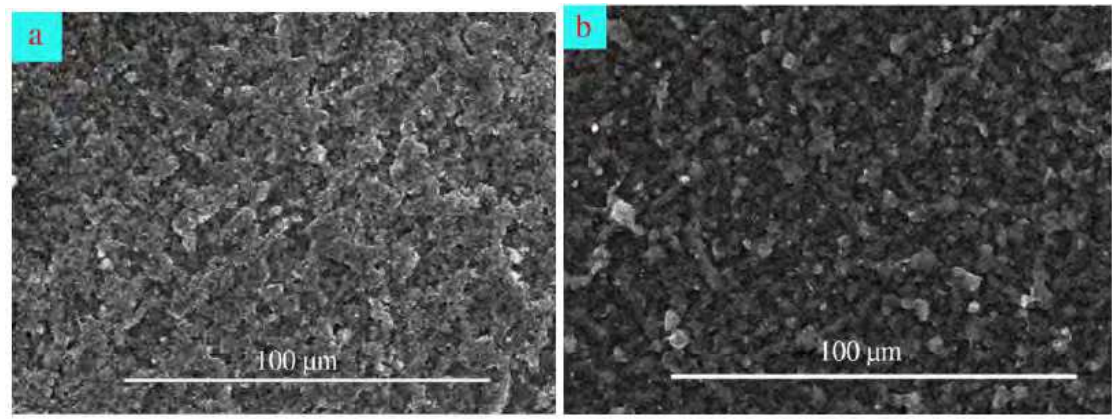

Fig. 16. SEM images of HMMT film surface (a) and NMMT film surface (b). 
The surface of the HMMT film is relatively flat (Fig.16a). The clay platelets seem to overlap each other, glued together by the polymer deposited and intercalated between them, in comparison with the insular pattern characteristic of the NMMT film surface (Fig. 5b). Additionally, compliant clay platelets possess the capability of covering the defects of the underlying layers, called "self-healing" by Kleinfeld and Ferguson (Kleinfeld \& Ferguson, 1994). This is why we used some deformed plates to illustrate clay platelets in Fig.12.

\subsubsection{Four important roles played by AAER}

What is worth our special attention are the four important roles that AAER play in the whole process, from intercalation to assembly.

Firstly, AAER serves as the intercalation agent in the hydrothermal process, ensuring the nano-laminated structure. Owing to its hydroxyl, carboxyl, epoxy groups and benzene rings introduced into the polymer molecule during polymerization, AAER possesses excellent compatibility with various materials as well as mechanical flexibility and strength. This is the first reason why we employ AAER as the organic component in this composite system.

Secondly, part of the deposited AAER from suspensions acts as the mortar in the z-direction around intercalated or non-intercalated platelets. This is evident in the TGA results of HMMT powder and HMMT film. The total organic content in HMMT films can be adjusted from $\sim 5 \mathrm{wt} \%$ to $\sim 15 \mathrm{wt} . \%$, depending on the mass ratio of NMMT to AAER in aqueous suspensions. In comparison, the organic content in HMMT powder is always below $5 \mathrm{wt} . \%$.

Thirdly, intercalated AAER contributes to more stable suspension for EPD. Proof is provided by the Zeta potential data: -36.69 and $-63.29 \mathrm{mV}$ for the aqueous suspension of NMMT and HMMT powder respectively. Furthermore, according to Scherrer's equation, from broadening of $(00 \mathrm{~L})$ peaks in XRD patterns, we can estimate the size of ordered domains in the direction normal to the substrate surface: $23.4 \mathrm{~nm}$ for the NMMT film (approximately 18 NMMT platelets piled together) and the $11.3 \mathrm{~nm}$ for heat-treated HMMT film (approximately 8 pieces of HMMT platelets piled together).

Finally, the electrophoretic capability of HMMT powder in water was greater than that of SMMT powder, characterized by the conductivity: 0.140 and $0.067 \mathrm{mS} / \mathrm{cm}$ for the aqueous suspension of HMMT and SMMT powder respectively. This explains why the film prepared with SMMT powder is much thinner than that prepared with HMMT powder by EPD from aqueous suspension of the same mass concentration.

\subsubsection{Enhanced mechanical property of the films}

Nano-indentation measurements were performed on NMMT film, AAER film and HMMT film prepared by the typical process stated in the experimental section (with $\sim 8$ wt.\% polymer in the composite, determined by TGA). Results show an increase in reduced

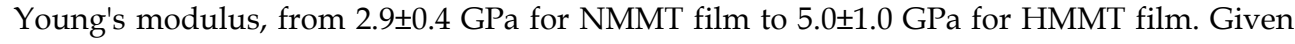
the low polymer content contained in the composite, this modulus enhancement is outstanding, which should be mainly attributed to the brick-and-mortar structure that plays a pivotal role in absorbing energy during elastic deformation of composite. As for the comparison between the mechanical property of the nanocomposite film constructed in this research and that of natural nacre, some points should be made here. 
First, given the difference between testing methods, the reduced Young's modulus above cannot be directly compared with Young's modulus ( 48.5 GPa) of nacre in the recent threepoint bend test.

Second, in addition to ordered layered structure, interfacial compatibility of the organic and inorganic components is a key factor. From this aspect, only certain types of polymer are effective in dramatically enhancing mechanical properties of such composite films. Thus, whether AAER is most suitable or not is still unknown.

Third, the thicknesses of organic layers and inorganic layers in our nano-laminated films are much thinner than those in nacre. In natural nacres, the biopolymer layers are usually 10-50 $\mathrm{nm}$ thick, providing necessary space for tight folding of polymer chains and certain degree of cross-linking of polymer. In comparison, in our laminated structure, polymer is confined within the interlayer space of smaller than $2 \mathrm{~nm}$. Thus, the degree of cross-linking of AAER with its percentage in total organic content is probably low, consistent with the result that no distinction in FTIR spectra and XRD patterns were observed between the as-deposited HMMT film and the heat-treated HMMT film. Meanwhile, aragonite layers in nacre are 200-900 nm thick, hundreds of times thicker than the clay layers in our film. This may well explain why natural nacre adopts the micro/nano composite structure but not the nano/nano composites structure. Research on the preparation of micro/nano laminated organic-inorganic composites is being conducted by our group.

Fourth, properties of clay platelets are fairly different with aragonite. Clay platelets are extremely compliant, while aragonite is much more rigid. Additionally, $\mathrm{CaCO}_{3}$ blocks have nano asperities that are about $30 \sim 100 \mathrm{~nm}$ in diameter, $10 \mathrm{~nm}$ in amplitude, providing additional friction when one block is sliding on the other.

\subsection{Summary}

The special assembly method-hydrothermal-electrophoretic assembly was successfully developed to prepare AAER/MMT nanocomposites that mimic nacre, both in structure and composition. The thickness of the nanocomposites film is controllable and can reach to more than $20 \mu \mathrm{m}$.

In this process, AAER plays four important roles as: intercalation agent in the hydrothermal process, binder around intercalated or non-intercalated platelets, stabilizing agent for MMT suspension, and improving the electric conductivity of MMT by AAER-intercalated.

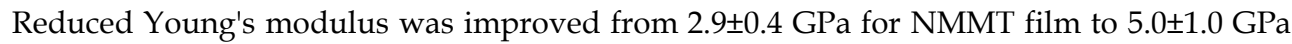
for HMMT film even at a low polymer content contained in the composite. The brick-andmortar nacre-like structure is mainly attributed to the improved mechanical properties by incorporating extra energy-absorbing mechanisms during elastic deformation.

\section{Conclusions}

This chapter has summarized three processes that can produce laminated biomimetic nanocomposites. The high-speed centrifugal process can produce nanocomposites up to a thickness of $200 \mu \mathrm{m}$ within minutes. The thick films produced have similar organic content and mechanical properties compared to that of lamella bones. The electrophoretic 
deposition of monomers and intercalated montmorillonite clay followed by ultraviolet initiated polymerization can produce dense laminated nano-composite films up to tens of $\mu \mathrm{m}$. The composite film exhibits four-fold improvement in Young's modulus and hardness over monolithic polyacrylamide polymers. Electrophoretic deposition combining intercalated montmorillonite nano-plates and polyelectrolyte such as acrylic anodic electrophoretic resin (AAER) can produce nanocomposites with organic content of $5 \mathrm{wt} \%$ to $15 \mathrm{wt} \%$. The composites obtained have good uniformity and significant improvement in Young's modulus and strength over monolithic montmorillonite films. These methods hold promise to fabricate laminated biomimetic materials at increased deposition rate. With the development of synthetic hydroxyapatite nanoplates (Le et al, 2009), these methods will enable the fabrication of a new generation of biomimetic nanocomposites for bone substitutes. This is becoming an area of great interest to clinicians as well as materials scientists.

\section{References}

Bonfield, W.; Wang, M. \& Tanner, K. E. (1998). Interfaces in analogue biomaterials. Acta Mater., 46 (7): 2509-2518

Chen, K. Y.; Wang, C. A.; Huang, Y. \& Lin, W. Preparation and characterization of polymerclay nanocomposite films, Science in China Series B: Chemistry, in press

Chen, R. F.; Wang, C. A.; Huang, Y. \& Le, H. R. (2008). An efficient biomimetic process for fabrication of artificial nacre with ordered-nanostructure. Mater. Sci. Eng. C, 28 (2): 218-222

Chen, X.; Sun, X. M. \& Li, Y. D. (2002). Self-assembling vanadium oxide nanotubes by organic molecular templates. Inorg. Chem., 41 (17): 4524-4530

Clegg, W. J.; Kendlaa, K.; Alford, N. M.; Button, T. W. \& Birchal, J. D. (1990). A simple way to make tough ceramics. Nature, 347 (6292) :455-457

Deville, S.; Saiz, E; Nalla, R. K. \& Tomsia, A. P. (2006). Freezing as a path to build complex composites. Science, 311 (5760): 515-518

Evans, A. G.; Suo, Z.; Wang, R. Z.; Aksay, I. A.; He, M. Y. \& Hutchinson, J. W. (2001). Model for the robust mechanical behavior of nacre. J. Mater. Res., 16 (9): 2475-2484

Fan, X.; Lochlin, J.; Youk, J.H.; Blanton, W.; Xia, C. \& Advincula, R. (2002). Nanostructured sexithiophene/clay hybrid mutilayers: a comparative structural and morphological characterization. Chem. Mater., 14 (5): 2184-2191

Fendler, J. H. (1996). Self-assembled nanostructured materials. Chem. Mater., 8(8):1616-1624

Graham, J. S.; Rosseinsky, D. R.; Slocombe, J. D.; Barrett, S. \& Francis, S. R. (1995). Electrochemistry of clay electrodeposition from sols: electron-transfer, deposition and microgravimetry studies. Colloid Surface A, 94(2-3): 177-188

Huang, M. H.; Dunn, B. S.; Soyez, H. \& Zink, J. I. (1998). In Situ probing by fluorescence spectroscopy of the formation of continuous highlyordered lamellar-phase mesostructured thin films. Langmuir, 14 (26): 7331-7333

Kleinfeld, E. R. \& Ferguson, G. S. (1994). Stepwise formation of multilayered nanostructural films from macromolecular precursors. Science, 265 (5170): 370-372

Kleinfeld, E. R. \& Ferguson, G.S. (1996). Healing of defects in the stepwise formation of polymer/silicate multilayer films. Chem. Mater., 8 (8): 1575-1778 
Kotov, N. A.; Haraszti, T.; Turi, L.; Zavala, G.; Geer, R. E.; Dekany, I. \& Fendler, J. H. (1997). Mechanism of and defect formation in the self-assembly of polymeric polycationmontmorillonite ultrathin films. J. Am. Chem. Soc., 119 (29): 6821-6832

Lan, T.; Kaviratna, P. D. \& Pinnavaia, T. J. (1994). On the nature of polyimide-clay hybrid composites. Chem. Mater., 6 (5): 573-575

Le, H. R.; Pranti-Haran, S.; Donnelly, K. and Keatch, R. P. (2009). Microstructure and Cell Adhesion of Hydroxyapatite/Collagen Composites. Proceedings of $11^{\text {th }}$ International Congress of the IUPESM, Sept 7-12, 2009, Munich, Germany.

Lin, W.; Wang, C. A.; Le, H. R.; Long, B. \& Huang, Y. (2008). Special assembly of laminated nanocomposite that mimics nacre. Mater. Sci. Eng. C, 28 (7): 1031-1037

Lin, W.; Wang, C. A.; Long, B. \& Huang, Y. (2008). Preparation of polymer-clay nanocomposite films by water-based electrodeposition. Compos. Sci Technol., 68 (34): 880-887

Long, B.; Wang, C. A.; Lin, W.; Huang, H. \& Sun, J. L. (2007). Polyacrylamide-clay nacre-like nanocomposites prepared by electrophoretic deposition. Compos. Sci Technol., 67 (13) $2770-2774$

Lu, Y. F.; Ganguli, R.; Drewien, C. A.; Anderson,M. T.; Brinker, C. J. ; Gong, W. L.; Guo, Y. X.; Soyez, H.; Dunn, B.; Huang, M. H. \& Zink, J. I. (1997). Continuous formation of supported cubic and hexagonal mesoporous films by sol-gel dip-coating. Nature, 389 (6649): 364-368

Sellinger, A.; Weiss, P. M.; Nguyen, A.; Lu, Y. F.; Assink, R. A.; Gong, W. \& Brinker, C. J. (1998). Continuous self-assembly of organic-inorganic nanocomposite coatings that mimic nacre. Nature, 394 (6690): 256-260

Smith, B. L.; Schaffer, T. E.; Viani, M.; Thompson, J. B.; Frederick, N. A.; Kindt, J.; Belcher, A.; Stucky, G. D.; Morse, D. E. \& Hansma, P. K. (1999). Molecular mechanistic origin of the toughness of natural adhesives, fibres and composites, Nature, 399 (6738): 761763

Tang, Z. Y.; Kotov, N. A.; Magonov, S. \& Ozturk, B. (2003). Nanostructured artificial nacre. Nature Mater., 2 (6): 413-418

Wang, C. A.; Huang, Y.; Zan, Q. F.; Zou, L. H. \& Cai, S. Y. (2002). Control of composition and structure in laminated silicon nitride/boron nitride composites. J. Am. Ceram. Soc., 85 (10): 2457-2461

Wang, R. Z.; Suo, Z.; Evans, A. G.; Yao, N. \& Aksay, I. A. (2001). Deformation mechanism in nacre. J. Mater. Res., 16 (9): 2485-2493

Wang, X. \& Li, Y. D. (2002). Selected-control hydrothermal synthesis of $\alpha$ - and $\beta-\mathrm{MnO}_{2}$ single crystal nanowires, J. Am. Chem. Soc., 124 (12): 2880-2881 


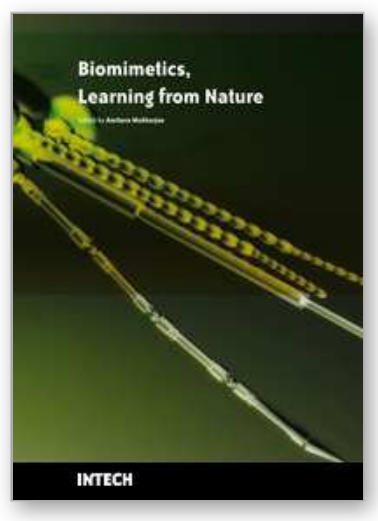

\section{Biomimetics Learning from Nature}

Edited by Amitava Mukherjee

ISBN 978-953-307-025-4

Hard cover, 534 pages

Publisher InTech

Published online 01, March, 2010

Published in print edition March, 2010

Nature's evolution has led to the introduction of highly efficient biological mechanisms. Imitating these mechanisms offers an enormous potential for the improvement of our day to day life. Ideally, by bio-inspiration we can get a better view of nature's capability while studying its models and adapting it for our benefit. This book takes us into the interesting world of biomimetics and describes various arenas where the technology is applied. The 25 chapters covered in this book disclose recent advances and new ideas in promoting the mechanism and applications of biomimetics.

\section{How to reference}

In order to correctly reference this scholarly work, feel free to copy and paste the following:

Chang-An Wang, Huirong Le and Yong Huang (2010). Rapid Assembly Processes of Ordered Inorganic/Organic Nanocomposites, Biomimetics Learning from Nature, Amitava Mukherjee (Ed.), ISBN: 978953-307-025-4, InTech, Available from: http://www.intechopen.com/books/biomimetics-learning-fromnature/rapid-assembly-processes-of-ordered-inorganic-organic-nanocomposites

\section{INTECH}

open science | open minds

\section{InTech Europe}

University Campus STeP Ri

Slavka Krautzeka 83/A

51000 Rijeka, Croatia

Phone: +385 (51) 770447

Fax: +385 (51) 686166

www.intechopen.com

\section{InTech China}

Unit 405, Office Block, Hotel Equatorial Shanghai

No.65, Yan An Road (West), Shanghai, 200040, China

中国上海市延安西路65号上海国际贵都大饭店办公楼 405 单元

Phone: +86-21-62489820

Fax: +86-21-62489821 
(C) 2010 The Author(s). Licensee IntechOpen. This chapter is distributed under the terms of the Creative Commons Attribution-NonCommercialShareAlike-3.0 License, which permits use, distribution and reproduction for non-commercial purposes, provided the original is properly cited and derivative works building on this content are distributed under the same license. 